JOURNAL OF THE

AMERICAN MATHEMATICAL SOCIETY

Volume 16, Number 4, Pages 901-915

S 0894-0347(03)00429-6

Article electronically published on May 1, 2003

\title{
GROMOV-WITTEN INVARIANTS ON GRASSMANNIANS
}

\author{
ANDERS SKOVSTED BUCH, ANDREW KRESCH, AND HARRY TAMVAKIS
}

\section{INTRODUCTION}

The central theme of this paper is the following result: any three-point genus zero Gromov-Witten invariant on a Grassmannian $X$ is equal to a classical intersection number on a homogeneous space $Y$ of the same Lie type. We prove this when $X$ is a type $A$ Grassmannian, and, in types $B, C$, and $D$, when $X$ is the Lagrangian or orthogonal Grassmannian parametrizing maximal isotropic subspaces in a complex vector space equipped with a non-degenerate skew-symmetric or symmetric form. The space $Y$ depends on $X$ and the degree of the Gromov-Witten invariant considered. For a type $A$ Grassmannian, $Y$ is a two-step flag variety, and in the other cases, $Y$ is a sub-maximal isotropic Grassmannian.

Our key identity for Gromov-Witten invariants is based on an explicit bijection between the set of rational maps counted by a Gromov-Witten invariant and the set of points in the intersection of three Schubert varieties in the homogeneous space $Y$. The proof of this result uses no moduli spaces of maps and requires only basic algebraic geometry. It was observed in [Bu1] that the intersection and linear span of the subspaces corresponding to points on a curve in a Grassmannian, called the kernel and span of the curve, have dimensions which are bounded below and above, respectively. The aforementioned bijection sends a rational map $\mathbb{P}^{1} \rightarrow X$ to the pair consisting of the kernel and span of its image. (In types $B, C$, and $D$, the span is the orthogonal complement of the kernel and can therefore be disregarded.)

The first application is in type $A$ : our main theorem implies that the problem of finding a positive combinatorial formula for the above Gromov-Witten invariants on Grassmannians is a special case of the problem of providing such a formula for the classical structure constants on flag varieties. Both of these open questions have received much attention in recent years in the contexts of quantum cohomology $[\mathrm{Be},[\mathrm{BCF}]$, representation theory $[\mathrm{BCM}],[\mathrm{BKMW}], \mathrm{BMW}], \mathrm{Tu}]$, and Schubert calculus [BS], [FK], [S]. The present connection becomes particularly interesting due to a conjecture of Knutson for the classical structure constants [K]. While this conjecture is known to be false in general, we have strong computational evidence suggesting that the conjecture is true in the special case of two-step flag varieties. Thus Knutson's conjecture for two-step flags specializes to a conjectural quantum Littlewood-Richardson rule.

Received by the editors July 18, 2002.

2000 Mathematics Subject Classification. Primary 14N35; Secondary 14M15, 14N15, 05E15.

Key words and phrases. Gromov-Witten invariants, Grassmannians, Flag varieties, Schubert varieties, Quantum cohomology, Littlewood-Richardson rule.

The authors were supported in part by NSF Grant DMS-0070479 (Buch), an NSF Postdoctoral Research Fellowship (Kresch), and NSF Grant DMS-0296023 (Tamvakis). 
In types $B, C$, and $D$, our result is used to give new proofs of the structure theorems for the quantum cohomology of Lagrangian and orthogonal Grassmannians (denoted $L G$ and $O G$ ) which were recently obtained in [KT1] and [KT2]. The strategy here is to employ Schubert calculus on isotropic Grassmannians to compute a few key Gromov-Witten invariants. Combining this with some basic geometric results from the aforementioned papers, we deduce the quantum Pieri and Giambelli formulas for $L G$ and $O G$. The earlier proofs relied on an analysis of special loci on certain Quot scheme compactifications of the moduli space of degree $d$ rational maps to either space. In contrast, the arguments provided here avoid the use of sophisticated moduli space constructions.

The methods of this article can also be used to prove a quantum Pieri rule for the quantum cohomology of sub-maximal isotropic Grassmannians. This application will be presented in BKT2].

Our paper is organized by treating different Lie types in different sections. Type $A$ Grassmannians are described in Section 2, Lagrangian Grassmannians (type $C$ ) in Section 3, and orthogonal Grassmannians (types $B$ and $D$ ) in Section 4 .

The authors wish to thank Michel Brion, Allen Knutson, and Laurent Manivel for helpful discussions related to this work.

\section{The Grassmannian $G(k, n)$}

2.1. Preliminaries. We review some standard definitions related to intersection theory on Grassmannians; see $[\mathrm{F}]$ for a detailed exposition. Let $X=G(k, E)=$ $G(k, n)$ be the Grassmannian of $k$-dimensional subspaces of $E=\mathbb{C}^{n}$, and set $r=$ $n-k$. Given a fixed full flag $F_{1} \subset F_{2} \subset \cdots \subset F_{n}=E$ and a partition $\lambda=\left(\lambda_{1} \geqslant\right.$ $\left.\lambda_{2} \geqslant \cdots \geqslant \lambda_{k}\right)$ with $\lambda_{1} \leqslant r$ there is the Schubert variety

$$
X_{\lambda}\left(F_{\bullet}\right)=\left\{V \in X \mid \operatorname{dim}\left(V \cap F_{r+i-\lambda_{i}}\right) \geqslant i, \forall 1 \leqslant i \leqslant k\right\} .
$$

The codimension of this variety is the weight $|\lambda|=\sum \lambda_{i}$ of $\lambda$. We let $\sigma_{\lambda}$ denote the class of $X_{\lambda}\left(F_{\bullet}\right)$ in the cohomology ring $H^{*}(X ; \mathbb{Z})$.

A rational map of degree $d$ to $X$ is a morphism $f: \mathbb{P}^{1} \rightarrow X$ such that

$$
\int f_{*}\left[\mathbb{P}^{1}\right] \cdot \sigma_{1}=d
$$

i.e. $d$ is the number of points in $f^{-1}\left(X_{1}\left(F_{\bullet}\right)\right)$ when $F_{\bullet}$ is in general position. All the Gromov-Witten invariants considered in this paper are three-point and genus zero. Given a degree $d \geqslant 0$ and partitions $\lambda, \mu$, and $\nu$ such that $|\lambda|+|\mu|+|\nu|=k r+n d$, we define the Gromov-Witten invariant $\left\langle\sigma_{\lambda}, \sigma_{\mu}, \sigma_{\nu}\right\rangle_{d}$ to be the number of rational maps $f: \mathbb{P}^{1} \rightarrow X$ of degree $d$ such that $f(0) \in X_{\lambda}\left(F_{\bullet}\right), f(1) \in X_{\mu}\left(G_{\bullet}\right)$, and $f(\infty) \in X_{\nu}\left(H_{\bullet}\right)$, for given flags $F_{\bullet}, G_{\bullet}$, and $H_{\bullet}$ in general position.

The next proposition implies that the Gromov-Witten invariant which counts degree $d$ maps to the Grassmannian $G(d, 2 d)$ through three general points, is equal to 1 . We will say that two points $U, V$ of $G(d, 2 d)$ are in general position if the intersection $U \cap V$ of the corresponding subspaces is trivial.

Proposition 1. Let $U, V$, and $W$ be three points of $\mathcal{Z}=G(d, 2 d)$ which are pairwise in general position. Then there is a unique morphism $f: \mathbb{P}^{1} \rightarrow \mathcal{Z}$ of degree $d$ such that $f(0)=U, f(1)=V$, and $f(\infty)=W$.

Proof. Let $U, V$, and $W$ be given, in pairwise general position. Given a basis $\left\{v_{1}, \ldots, v_{d}\right\}$ of $V$ we can construct a morphism $f: \mathbb{P}^{1} \rightarrow \mathcal{Z}$ of degree $d$ such 
that $f(0)=U, f(1)=V$, and $f(\infty)=W$ as follows. For $1 \leqslant i \leqslant d$ we let $u_{i} \in U$ and $w_{i} \in W$ be the projections of $v_{i} \in \mathbb{C}^{2 d}=U \oplus W$. Then $f(s: t)=\operatorname{Span}\left\{s u_{1}+t w_{1}, \ldots, s u_{d}+t w_{d}\right\}$ satisfies the required conditions, where $(s: t)$ are the homogeneous coordinates on $\mathbb{P}^{1}$. Furthermore, one checks easily that the map $f$ does not depend on the choice of basis of $V$.

Now suppose $f: \mathbb{P}^{1} \rightarrow \mathcal{Z}$ is any morphism of degree $d$ which sends $0,1, \infty$ to $U, V, W$, respectively. Let $S \subset \mathbb{C}^{2 d} \otimes \mathcal{O}_{\mathcal{Z}}$ be the tautological bundle on $\mathcal{Z}$. The general position hypothesis implies that $f^{*} S=\mathcal{O}(-1)^{\oplus d}$. Therefore we can write

$$
f(s: t)=\operatorname{Span}\left\{s u_{1}+t w_{1}, \ldots, s u_{d}+t w_{d}\right\}
$$

for suitable vectors $u_{i}, w_{i} \in \mathbb{C}^{2 d}$. It follows that $f$ is the map constructed as above from the basis $\left\{v_{1}, \ldots, v_{d}\right\}$, where $v_{i}=u_{i}+w_{i}$.

Let $C$ be a non-empty subset of $X$. Define the kernel of $C$ to be the intersection of all the subspaces $V \subset E$ corresponding to points of $C$. Similarly, the span of $C$ is the linear span of these subspaces.

$$
\operatorname{Ker}(C)=\bigcap_{V \in C} V ; \quad \operatorname{Span}(C)=\sum_{V \in C} V .
$$

The kernel and span of a rational map $f: \mathbb{P}^{1} \rightarrow X$ are defined to be the kernel and span, respectively, of the image of $f$. By considering the splitting type of the pullback of the tautological subbundle, one proves (see [Bu1, Lemma 1]) that the kernel of $f$ (resp. the span of $f$ ) has dimension at least $k-d$ (resp. at most $k+d$ ) when $f$ has degree $d$. In fact the main point of [Bu1] is to use the span of a rational map to obtain greatly simplified proofs of type $A$ results such as the quantum Giambelli and Pieri formulas, first proved by Bertram [Be].

2.2. Main result for $G(k, n)$. Let $X=G(k, n)$, and fix $E=\mathbb{C}^{n}$ as before. Given integers $a$ and $b$, we let $F(a, b ; E)=F(a, b ; n)$ denote the two-step flag variety parametrizing pairs of subspaces $(A, B)$ with $A \subset B \subset E, \operatorname{dim} A=a$ and $\operatorname{dim} B=$ $b$. (This variety is empty unless $0 \leqslant a \leqslant b \leqslant n$, and when non-empty, has dimension $(n-b) b+(b-a) a$.) For any non-negative integer $d$ we set $Y_{d}=F(k-d, k+d ; E)$. Our main result will be used to identify Gromov-Witten invariants on $X$ with classical intersection numbers on the flag varieties $Y_{d}$.

To any subvariety $\mathcal{W} \subset X$ we associate the following subvariety in $Y_{d}$ :

$$
\mathcal{W}^{(d)}=\left\{(A, B) \in Y_{d} \mid \exists V \in \mathcal{W}: A \subset V \subset B\right\} .
$$

Let $F(k-d, k, k+d ; E)$ denote the variety of three-step flags in $E$ of the indicated dimensions and $\pi_{1}: F(k-d, k, k+d ; E) \rightarrow X$ and $\pi_{2}: F(k-d, k, k+d ; E) \rightarrow Y_{d}$ the natural projections. We have $\mathcal{W}^{(d)}=\pi_{2}\left(\pi_{1}^{-1}(\mathcal{W})\right)$; moreover, if $\mathcal{W}=X_{\lambda}\left(F_{\bullet}\right)$ is a Schubert variety in $X$, then $\mathcal{W}^{(d)}=X_{\lambda}^{(d)}\left(F_{\bullet}\right)$ is a Schubert variety in $Y_{d}$ (we describe this Schubert variety in more detail after Corollary I). Since the fibers of $\pi_{2}$ are isomorphic to $G(d, 2 d)$, the codimension of $X_{\lambda}^{(d)}\left(F_{\bullet}\right)$ in $Y_{d}$ is at least $|\lambda|-d^{2}$. Notice also that $\operatorname{dim} Y_{d}=k r+n d-3 d^{2}$.

Theorem 1. Let $\lambda, \mu$, and $\nu$ be partitions and $d$ an integer such that $|\lambda|+|\mu|+|\nu|=$ $k r+n d$, and let $F_{\bullet}, G_{\bullet}$, and $H_{\bullet}$ be complete flags of $E=\mathbb{C}^{n}$ in general position. Then the map $f \mapsto(\operatorname{Ker}(f)$, $\operatorname{Span}(f))$ gives a bijection of the set of rational maps $f: \mathbb{P}^{1} \rightarrow G(k, n)$ of degree d satisfying $f(0) \in X_{\lambda}\left(F_{\bullet}\right), f(1) \in X_{\mu}\left(G_{\bullet}\right)$, and $f(\infty) \in$ 
$X_{\nu}\left(H_{\bullet}\right)$, with the set of points in the intersection $X_{\lambda}^{(d)}\left(F_{\bullet}\right) \cap X_{\mu}^{(d)}\left(G_{\bullet}\right) \cap X_{\nu}^{(d)}\left(H_{\bullet}\right)$ in $Y_{d}=F(k-d, k+d ; n)$.

Proof. Let $f: \mathbb{P}^{1} \rightarrow X$ be a rational map as in the statement of the theorem. The following dimension-counting argument shows that $d \leqslant \min (k, n-k)$, and moreover, $\operatorname{dim} \operatorname{Ker}(f)=k-d$ and $\operatorname{dim} \operatorname{Span}(f)=k+d$. Let $a=\operatorname{dim} \operatorname{Ker}(f)$ and $b=\operatorname{dim} \operatorname{Span}(f)$. In the two-step flag variety $Y^{\prime}=F(a, b ; E)$ there are associated Schubert varieties $X_{\lambda}^{\prime}\left(F_{\bullet}\right), X_{\mu}^{\prime}\left(G_{\bullet}\right)$, and $X_{\nu}^{\prime}\left(H_{\bullet}\right)$, defined as in (1). If we write $e_{1}=k-a$ and $e_{2}=b-k$, then the codimension of $X_{\lambda}^{\prime}$ in $Y^{\prime}$ is at least $|\lambda|-e_{1} e_{2}$, and similar inequalities hold with $\mu$ and $\nu$ in place of $\lambda$. Since $(\operatorname{Ker}(f), \operatorname{Span}(f)) \in$ $X_{\lambda}^{\prime}\left(F_{\bullet}\right) \cap X_{\mu}^{\prime}\left(G_{\bullet}\right) \cap X_{\nu}^{\prime}\left(H_{\bullet}\right)$ and the three flags are in general position, we obtain $k r+d n-3 e_{1} e_{2} \leqslant \operatorname{dim} F(a, b ; E)$, and therefore

$$
d n \leqslant e_{1} a+e_{2}(n-b)+2 e_{1} e_{2} .
$$

We know that $e_{1} \leqslant d$ and $e_{2} \leqslant d$, and hence that the right-hand side of (2) is at most $d(a+n-b)+2 e_{1} e_{2}$. It follows that $\left(e_{1}+e_{2}\right)^{2} \leqslant 2 d\left(e_{1}+e_{2}\right) \leqslant 4 e_{1} e_{2}$, and therefore $e_{1}=e_{2}=d$.

Let $\mathcal{M}$ denote the set of rational maps in the statement of the theorem, and set $\mathcal{I}=X_{\lambda}^{(d)}\left(F_{\bullet}\right) \cap X_{\mu}^{(d)}\left(G_{\bullet}\right) \cap X_{\nu}^{(d)}\left(H_{\bullet}\right)$. If $f \in \mathcal{M}$ then $(\operatorname{Ker}(f), \operatorname{Span}(f)) \in \mathcal{I}$. It remains to show that for any point $(A, B) \in \mathcal{I}$ there is a unique map $f \in \mathcal{M}$ such that $\operatorname{Ker}(f)=A$ and $\operatorname{Span}(f)=B$.

Consider the three-step flag variety $Y^{\prime \prime}=F(k-d, k-d+1, k+d ; E)$ and the projection $\pi: Y^{\prime \prime} \rightarrow Y_{d}$. Note that $\operatorname{dim} Y^{\prime \prime}=\operatorname{dim} Y_{d}+2 d-1$. To each subvariety $\mathcal{W} \subset G(k, E)$ we associate $\mathcal{W}^{\prime \prime} \subset Y^{\prime \prime}$ defined by

$$
\mathcal{W}^{\prime \prime}=\left\{\left(A, A^{\prime}, B\right) \in Y^{\prime \prime} \mid \exists V \in \mathcal{W}: A^{\prime} \subset V \subset B\right\} .
$$

We find that the codimension of $X_{\mu}^{\prime \prime}\left(G_{\bullet}\right)$ in $Y^{\prime \prime}$ is at least $|\mu|-d^{2}+d$, and similarly for $X_{\nu}^{\prime \prime}\left(H_{\bullet}\right)$. Since the three flags are in general position, and $\pi^{-1}\left(X_{\lambda}^{(d)}\left(F_{\bullet}\right)\right)$ has codimension at least $|\lambda|-d^{2}$ in $Y^{\prime \prime}$, we have

$$
\pi^{-1}\left(X_{\lambda}^{(d)}\left(F_{\bullet}\right)\right) \cap X_{\mu}^{\prime \prime}\left(G_{\bullet}\right) \cap X_{\nu}^{\prime \prime}\left(H_{\bullet}\right)=\emptyset,
$$

and the same is true for the other two analogous triple intersections.

Given $(A, B) \in \mathcal{I}$, we let $\mathcal{Z}=G(d, B / A) \subset X$ be the set of $k$-planes in $E$ between $A$ and $B$. Then $X_{\lambda}\left(F_{\bullet}\right) \cap \mathcal{Z}, X_{\mu}\left(G_{\bullet}\right) \cap \mathcal{Z}$, and $X_{\nu}\left(H_{\bullet}\right) \cap \mathcal{Z}$ are non-empty Schubert varieties in $\mathcal{Z}$. Choose three points $U, V$, and $W$ in $\mathcal{Z}$, one from each intersection; the equations (3) show that these three points are in pairwise general position. Observe that any positive dimensional Schubert variety in $\mathcal{Z}$ must contain a point $U^{\prime}$ which meets $U$ non-trivially, and similarly for $V$ and $W$. We deduce that each of $X_{\lambda}\left(F_{\bullet}\right) \cap \mathcal{Z}, X_{\mu}\left(G_{\bullet}\right) \cap \mathcal{Z}$, and $X_{\nu}\left(H_{\bullet}\right) \cap \mathcal{Z}$ must be a single point. Proposition 1 now supplies the unique $f: \mathbb{P}^{1} \rightarrow X$ in $\mathcal{M}$ with $\operatorname{Ker}(f)=A$ and $\operatorname{Span}(f)=B$.

Remark 1. One can rephrase Theorem 1 using rational curves in $X$, instead of rational maps to $X$. Indeed, the construction in Proposition 1 shows that every rational map $f$ that is counted in Theorem 1 is an embedding of $\mathbb{P}^{1}$ into $X$ of degree equal to the degree of the image of $f$. Notice also that the theorem implies that all of these maps have different images. 
It follows from Theorem 1 that we can express any Gromov-Witten invariant of degree $d$ on $G(k, n)$ as a classical intersection number on $Y_{d}=F(k-d, k+d ; n)$. Let $\left[X_{\lambda}^{(d)}\right] \in H^{*}\left(Y_{d}, \mathbb{Z}\right)$ denote the cohomology class of $X_{\lambda}^{(d)}\left(F_{\bullet}\right)$.

Corollary 1. Let $\lambda, \mu$, and $\nu$ be partitions and $d \geqslant 0$ an integer such that $|\lambda|+$ $|\mu|+|\nu|=k r+n d$. We then have

$$
\left\langle\sigma_{\lambda}, \sigma_{\mu}, \sigma_{\nu}\right\rangle_{d}=\int_{F(k-d, k+d ; n)}\left[X_{\lambda}^{(d)}\right] \cdot\left[X_{\mu}^{(d)}\right] \cdot\left[X_{\nu}^{(d)}\right] .
$$

The Schubert varieties in $F(a, b ; n)$ are indexed by permutations $w \in S_{n}$ with $w(i)<w(i+1)$ for all $i \notin\{a, b\}$. Given a full flag $F_{\bullet}$. in $E$, we define $X_{w}\left(F_{\bullet}\right) \subset$ $F(a, b ; n)$ as the locus of flags $A \subset B \subset E$ such that

$\operatorname{dim}\left(A \cap F_{i}\right) \geqslant \#\{p \leqslant a \mid w(p)>n-i\}$ and $\operatorname{dim}\left(B \cap F_{i}\right) \geqslant \#\{p \leqslant b \mid w(p)>n-i\}$ for all $i$. Recall that every partition $\lambda$ indexing a Schubert variety $X_{\lambda}\left(F_{\bullet}\right) \subset G(k, n)$ corresponds to a Grassmannian permutation $w=w_{\lambda} \in S_{n}$, determined by the relations $w(i)=\lambda_{k-i+1}+i$ for $i \leqslant k$ and $w(i)<w(i+1)$ for $i>k$. The permutation $w_{\lambda, d}$ associated to the modified Schubert variety $X_{\lambda}^{(d)}\left(F_{\bullet}\right)$ in $F(k-d, k+d ; n)$ is obtained from $w_{\lambda}$ by sorting the values $w(k-d+1), \ldots, w(k+d)$ to be in increasing order.

The proof of the next corollary gives a geometric explanation for Yong's upper bound for the $q$-degrees in a quantum product $[\mathrm{Y}]$.

Corollary 2 (Yong). In the situation of Corollary 1, if any of $\lambda_{d}, \mu_{d}$, and $\nu_{d}$ is less than $d$, then $\left\langle\sigma_{\lambda}, \sigma_{\mu}, \sigma_{\nu}\right\rangle_{d}=0$.

Proof. Observe that when $\lambda_{d}<d$, the codimension of $X_{\lambda}^{(d)}\left(F_{\bullet}\right)$ in $Y_{d}$ is strictly greater than $|\lambda|-d^{2}$. This follows by computing the length of the Weyl group element $w_{\lambda, d}$ associated to $X_{\lambda}^{(d)}\left(F_{\bullet}\right)$. Therefore, when any of $\lambda_{d}, \mu_{d}$, or $\nu_{d}$ is less than $d$, the sum of the codimensions of the three modified Schubert varieties which appear in the statement of Theorem 1 is greater than the dimension of $Y_{d}=F(k-d, k+d ; n)$.

Remark 2. For flag varieties $X$ other than Grassmannians, it is not in general true that the rational maps to $X$ counted by a Gromov-Witten invariant are distinguished by their kernels and spans (see $\mathrm{Bu} 2$ for definitions). For example, if $F_{\bullet}$, $G_{\bullet}$, and $H_{\bullet}$ are three general points in the complete flag variety $X=F(5)$, then there are exactly two rational maps of multidegree $(2,3,3,2)$ through these points, and they both have kernel $\left(0,0,0, F_{4} \cap G_{4} \cap H_{4}\right)$ and $\operatorname{span}\left(F_{1}+G_{1}+H_{1}, \mathbb{C}^{5}, \mathbb{C}^{5}, \mathbb{C}^{5}\right)$.

2.3. Knutson's conjecture for two-step flag varieties. We denote by $\left[X_{w}\right]$ the cohomology class of the Schubert variety $X_{w} \subset F(a, b ; n)$. Each indexing permutation $w$ corresponds to a string $J(w)$ of " 0 " s, " 1 "s, and " 2 "s of length $n$ : the positions of the "0"s (resp. "1"s) in $J(w)$ are recorded by $w(1), \ldots, w(a)$ (resp. $w(a+1), \ldots, w(b))$.

Define a puzzle to be a triangle decomposed into puzzle pieces of the types displayed below. Of these pieces the fourth and the sixth pieces come in different lengths. The fourth piece can have any number of "2"s (including none) to the right of the " 0 " on the top edge and equally many to the left of the "0" on the bottom edge. Similarly the sixth piece can have an arbitrary number of "0"s on the top and bottom edges. 


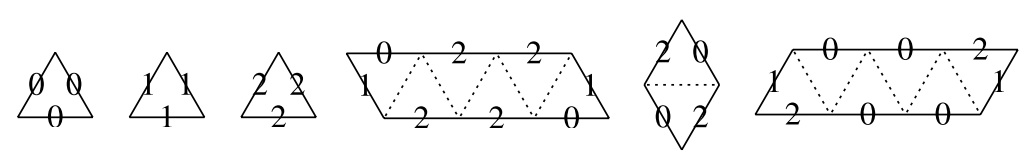

A puzzle piece may be rotated but not reflected when used in a puzzle. Furthermore, the common edges of two puzzle pieces next to each other must have the same labels. Figure 1 shows an example of a puzzle.

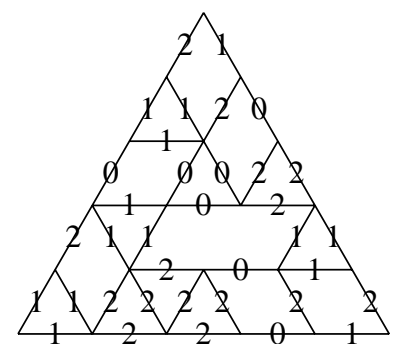

Figure 1. An example of a puzzle

We can now state Knutson's conjecture in the case of two-step flag varieties.

Conjecture 1 (Knutson). For any three Schubert varieties $X_{u}, X_{v}$, and $X_{w}$ in the flag variety $F(a, b ; n)$, the integral $\int_{F(a, b ; n)}\left[X_{u}\right] \cdot\left[X_{v}\right] \cdot\left[X_{w}\right]$ is equal to the number of puzzles such that $J(u), J(v)$, and $J(w)$ are the labels on the north-west, north-east, and south sides when read in clockwise order.

This conjecture has been verified for all two-step flag varieties $F(a, b ; n)$ for which $n \leqslant 16$. It is a special case of a general conjecture by Knutson for the Schubert structure constants on all partial flag varieties, which was circulated in the fall of 1999 [K]. Although the Grassmannian case of this conjecture has been proved KTW], Knutson quickly discovered counterexamples to the general conjecture. In fact, it fails for the three-step flag variety $F(1,3,4 ; 5)$.

2.4. A quantum Littlewood-Richardson rule. Let $X_{\lambda}\left(F_{\bullet}\right)$ be a Schubert variety in the Grassmannian $X=G(k, n)$ and fix a degree $d \geqslant 0$. The 012-string $J^{d}(\lambda)=J\left(w_{\lambda, d}\right)$ associated to the modified Schubert variety $X_{\lambda}^{(d)}\left(F_{\bullet}\right)$ in $Y_{d}=$ $F(k-d, k+d ; n)$ may be obtained as follows. Begin by drawing the Young diagram of the partition $\lambda$ in the upper-left corner of a $k \times r$ rectangle. On the path from the lower-left to the upper-right corner of this rectangle which follows the border of $\lambda$ we then put a label on each step. The leftmost $d$ horizontal steps are labeled "1", while the remaining $r-d$ horizontal steps are labeled "2". The top $d$ vertical steps are labeled "1", while the bottom $k-d$ vertical steps are labeled " 0 ". The string $J^{d}(\lambda)$ then consists of these labels in lower-left to upper-right order. We will write $X_{J^{d}(\lambda)}\left(F_{\bullet}\right)$ for $X_{\lambda}^{(d)}\left(F_{\bullet}\right)$.

Example 1. When $X=G(4,9), d=2$, and $\lambda=(4,4,3,1)$, we get $X_{\lambda}^{(2)}\left(F_{\bullet}\right)=$ $X_{J^{2}(\lambda)}\left(F_{\bullet}\right) \subset F(2,6 ; 9)$, where $J^{2}(\lambda)=101202112$. This is illustrated in Figure 2

When Conjecture $\square$ is combined with Corollary 1 we arrive at the following quantum Littlewood-Richardson rule. 


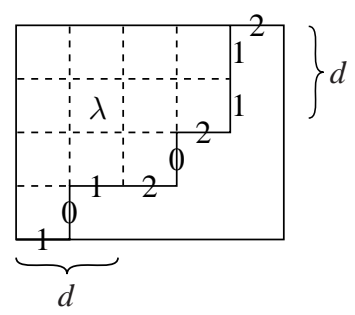

Figure 2. The 012-string associated to $\lambda$ and $d$

Conjecture 2. For partitions $\lambda, \mu, \nu$ such that $|\lambda|+|\mu|+|\nu|=k r+n d$ the GromovWitten invariant $\left\langle\sigma_{\lambda}, \sigma_{\mu}, \sigma_{\nu}\right\rangle_{d}$ is equal to the number of puzzles such that $J^{d}(\lambda)$, $J^{d}(\mu)$, and $J^{d}(\nu)$ are the labels on the north-west, north-east, and south sides when read in clockwise order.

The verified cases of Conjecture 1 imply that this quantum Littlewood-Richardson rule is true for all Grassmannians $G(k, n)$ for which $n \leqslant 16$. It has also been proved in some special cases including when $d=\min (k, r)$ or when $\lambda$ has length at most two or is a 'hook' partition. Furthermore, Conjecture 2 holds when $k \leqslant 3$. Details about this will be given in [BKT1]. See [Tu and references therein for alternative positive formulas for some of these special cases of Gromov-Witten invariants.

Example 2. Let $X=G(2,5), d=1$, and $Y_{1}=F(1,3 ; 5)$. We can then compute

$$
\left\langle\sigma_{(2,2)}, \sigma_{(2,1)}, \sigma_{(3,1)}\right\rangle_{1}=\int_{Y_{1}}\left[X_{12012}\right] \cdot\left[X_{10212}\right] \cdot\left[X_{10221}\right]=1 .
$$

The puzzle displayed in Figure 1 is the unique puzzle with the strings of these Schubert classes on its sides.

\section{The Lagrangian Grassmannian $L G(n, 2 n)$}

3.1. Preliminaries. In the following sections we will mostly adopt the notational conventions of [KT1] and [KT2]. We begin working with the Lagrangian Grassmannian $L G=L G(n, 2 n)$ parametrizing Lagrangian (i.e. maximal isotropic) subspaces of $E=\mathbb{C}^{2 n}$ equipped with a symplectic form. More generally, for each $k \leqslant n$, the Grassmannian $I G(k, 2 n)$ parametrizes $k$-dimensional isotropic subspaces in $E$. The dimension of the complex manifold $I G(k, 2 n)$ is $2 k(n-k)+k(k+1) / 2$.

Let $\mathcal{D}_{n}$ be the set of strict partitions $\lambda=\left(\lambda_{1}>\lambda_{2}>\cdots>\lambda_{\ell}>0\right)$ with $\lambda_{1} \leqslant n$. By convention, $\lambda_{j}=0$ for $j>\ell$. Fix an isotropic flag

$$
\text { F. : } 0 \subset F_{1} \subset F_{2} \subset \cdots \subset F_{n} \subset E
$$

of subspaces of $E$. For each $\lambda \in \mathcal{D}_{n}$ we have a codimension $|\lambda|$ Schubert variety $X_{\lambda}\left(F_{\bullet}\right) \subset L G$ defined as the locus of $V \in L G$ such that

$$
\operatorname{dim}\left(V \cap F_{n+1-\lambda_{i}}\right) \geqslant i, i=1, \ldots, \ell(\lambda) .
$$

Let $\sigma_{\lambda}$ be the class of $X_{\lambda}\left(F_{\bullet}\right)$ in the cohomology ring $H^{*}(L G, \mathbb{Z})$.

As in Section 2, a rational map to $L G$ means a morphism $f: \mathbb{P}^{1} \rightarrow L G$, and its degree is the degree of $f_{*}\left[\mathbb{P}^{1}\right] \cdot \sigma_{1}$. We also define the kernel of a subset of $L G$ as the intersection of the corresponding vector subspaces, and the kernel of a map 
$f$ to $L G$ as the kernel of its image (the span is the orthogonal complement of the kernel and is not necessary here). If $f$ has degree $d$ then $\operatorname{dim} \operatorname{Ker}(f) \geqslant n-d$. The following proposition is proved in the same way as its counterpart in type $A$.

Proposition 2. Let $U, V$, and $W$ be three points of $L G(d, 2 d)$ in pairwise general position. Then there is a unique morphism $f: \mathbb{P}^{1} \rightarrow L G(d, 2 d)$ of degree $d$ such that $f(0)=U, f(1)=V$, and $f(\infty)=W$.

Let $h(n, d)=(n+1)(n / 2+d)$. The Gromov-Witten invariant $\left\langle\sigma_{\lambda}, \sigma_{\mu}, \sigma_{\nu}\right\rangle_{d}$ is defined for $|\lambda|+|\mu|+|\nu|=h(n, d)$ and counts the number of rational maps $f: \mathbb{P}^{1} \rightarrow L G(n, 2 n)$ of degree $d$ such that $f(0) \in X_{\lambda}\left(F_{\bullet}\right), f(1) \in X_{\mu}\left(G_{\bullet}\right)$, and $f(\infty) \in X_{\nu}\left(H_{\bullet}\right)$, for given flags $F_{\bullet}, G_{\bullet}$, and $H_{\bullet}$ in general position. The next result for the three-point Gromov-Witten invariants of degree 1 on $L G$ was proved in [KT1, §3] via a geometric correspondence between lines on $L G(n, 2 n)$ and points in $L G(n+1,2 n+2)$ :

Proposition 3. For $\lambda, \mu, \nu \in \mathcal{D}_{n}$ we have

$$
\left\langle\sigma_{\lambda}, \sigma_{\mu}, \sigma_{\nu}\right\rangle_{1}=\frac{1}{2} \int_{L G(n+1,2 n+2)}\left[X_{\lambda}^{+}\right] \cdot\left[X_{\mu}^{+}\right] \cdot\left[X_{\nu}^{+}\right],
$$

where $X_{\lambda}^{+}, X_{\mu}^{+}, X_{\nu}^{+}$denote Schubert varieties in $L G(n+1,2 n+2)$.

3.2. Main result for $L G(n, 2 n)$. For any integer $d$ with $0 \leqslant d \leqslant n$, let $Y_{d}$ denote the isotropic Grassmannian $I G(n-d, 2 n)$. Given a subvariety $\mathcal{W} \subset L G$, we define $\mathcal{W}^{(d)} \subset Y_{d}$ by the prescription

$$
\mathcal{W}^{(d)}=\left\{A \in Y_{d} \mid \exists V \in \mathcal{W}: A \subset V\right\} .
$$

When $\mathcal{W}=X_{\lambda}\left(F_{\bullet}\right)$ is a Schubert variety in $L G$, we thus obtain a Schubert variety $X_{\lambda}^{(d)}\left(F_{\bullet}\right)$ in $Y_{d}$, of codimension at least $|\lambda|-d(d+1) / 2$. Note that $X_{\lambda}^{(1)}$ and $X_{\lambda}^{(2)}$ are the varieties which were denoted $\mathfrak{X}_{\lambda}^{\prime}$ and $\mathfrak{X}_{\lambda}^{\prime \prime}$, respectively, in [KT1, §3.1].

Theorem 2. Let $d$ be an integer and $\lambda, \mu, \nu \in \mathcal{D}_{n}$ be such that $|\lambda|+|\mu|+|\nu|=$ $h(n, d)$, and let $F_{\bullet}, G_{\bullet}$, and $H_{\bullet}$ be complete isotropic flags of $E=\mathbb{C}^{2 n}$ in general position. Then the map $f \mapsto \operatorname{Ker}(f)$ gives a bijection of the set of rational maps $f: \mathbb{P}^{1} \rightarrow L G(n, 2 n)$ of degree d satisfying $f(0) \in X_{\lambda}\left(F_{\bullet}\right), f(1) \in X_{\mu}\left(G_{\bullet}\right)$, and $f(\infty) \in X_{\nu}\left(H_{\bullet}\right)$, with the set of points in the intersection $X_{\lambda}^{(d)}\left(F_{\bullet}\right) \cap X_{\mu}^{(d)}\left(G_{\bullet}\right) \cap$ $X_{\nu}^{(d)}\left(H_{\bullet}\right)$ in $Y_{d}=I G(n-d, 2 n)$.

Proof. We proceed as in the proof of Theorem 1]. Given a map $f$ as in the statement of the theorem, set $e=n-\operatorname{dim} \operatorname{Ker}(f)$; we know that $e \leqslant d$. The codimension of $X_{\lambda}^{(e)}\left(F_{\bullet}\right)$ in $Y_{e}$ is at least $|\lambda|-e(e+1) / 2$, and similarly for $X_{\mu}^{(e)}\left(G_{\bullet}\right)$ and $X_{\nu}^{(e)}\left(H_{\bullet}\right)$. Since $\operatorname{Ker}(f) \in X_{\lambda}^{(e)}\left(F_{\bullet}\right) \cap X_{\mu}^{(e)}\left(G_{\bullet}\right) \cap X_{\nu}^{(e)}\left(H_{\bullet}\right)$, a dimension count shows that

$$
h(n, d)-3 e(e+1) / 2 \leqslant \operatorname{dim} I G(n-e, 2 n),
$$

which simplifies to $d \leqslant e$, and hence $\operatorname{dim} \operatorname{Ker}(f)=n-d$. Therefore, $\operatorname{Ker}(f) \in \mathcal{I}$, where $\mathcal{I}$ denotes the intersection in $Y_{d}$ from the statement of the theorem. The argument is completed as in type $A$, except that the type $C$ analogue of the variety $Y^{\prime \prime}$ is the isotropic partial flag space $I G(n-d, n-d+1 ; 2 n)$, and we invoke Proposition 2 instead of Proposition 1

Exactly as in type $A$, Theorem 2 identifies Gromov-Witten invariants on $L G$ with classical structure constants on isotropic Grassmannians. 
Corollary 3. Let $d \geqslant 0$ and choose $\lambda, \mu, \nu \in \mathcal{D}_{n}$ with $|\lambda|+|\mu|+|\nu|=h(n, d)$. We then have

$$
\left\langle\sigma_{\lambda}, \sigma_{\mu}, \sigma_{\nu}\right\rangle_{d}=\int_{I G(n-d, 2 n)}\left[X_{\lambda}^{(d)}\right] \cdot\left[X_{\mu}^{(d)}\right] \cdot\left[X_{\nu}^{(d)}\right] .
$$

Each $\lambda \in \mathcal{D}_{n}$ corresponds to a maximal Grassmannian element

$$
w_{\lambda}=\left(\bar{\lambda}_{1}, \ldots, \bar{\lambda}_{\ell}, \lambda_{n-\ell}^{\prime}, \ldots, \lambda_{1}^{\prime}\right)
$$

of the Weyl group $W_{n}$ for the root system $C_{n}$ (see [KT1, Sec. 2]). When the length $\ell$ of $\lambda$ is at least $d$, the Weyl group element $w_{\lambda, d}$ in $W_{n}$ associated to $X_{\lambda}^{(d)}\left(F_{\bullet}\right)$ is obtained from $w_{\lambda}$ by removing the bars from the first $d$ entries and sorting them to be in increasing order. In this case, the codimension of $X_{\lambda}^{(d)}\left(F_{\bullet}\right)$ in $I G(n-d, 2 n)$ is exactly $|\lambda|-d(d+1) / 2$.

The vanishing statement in next corollary was shown in [KT1, Prop. 10] using different methods; we argue here as in the proof of Corollary 2

Corollary 4. In the situation of Corollary [3, if any of $\lambda, \mu$, and $\nu$ has length less than $d$, then $\left\langle\sigma_{\lambda}, \sigma_{\mu}, \sigma_{\nu}\right\rangle_{d}=0$.

3.3. Quantum cohomology of $L G(n, 2 n)$. In this section, we use Corollaries 3 and 4 to give new proofs of the quantum Pieri rule [KT1 Prop. 8] and quantum Giambelli formula [KT1, Thm. 1] for $L G$. These are key ingredients used to describe the multiplicative structure of the quantum cohomology ring $Q H^{*}(L G(n, 2 n))$. Our proofs will require the crucial fact that the product defined in (6) is associative; a reference for this basic result is the expository paper [FP].

The (small) quantum cohomology ring $Q H^{*}(L G)$ is a $\mathbb{Z}[q]$-algebra which is isomorphic to $H^{*}(L G, \mathbb{Z}) \otimes_{\mathbb{Z}} \mathbb{Z}[q]$ as a module over $\mathbb{Z}[q]$. Here $q$ is a formal variable of degree $n+1$. The ring structure on $Q H^{*}(L G)$ is determined by the relation

$$
\sigma_{\lambda} \cdot \sigma_{\mu}=\sum\left\langle\sigma_{\lambda}, \sigma_{\mu}, \sigma_{\nu^{\prime}}\right\rangle_{d} \sigma_{\nu} q^{d}
$$

the sum over $d \geqslant 0$ and strict partitions $\nu$ with $|\nu|=|\lambda|+|\mu|-d(n+1)$. Here $\nu^{\prime}$ denotes the dual partition of $\nu$, defined so that the classes $\sigma_{\nu}$ and $\sigma_{\nu^{\prime}}$ are Poincaré dual to each other in $H^{*}(L G, \mathbb{Z})$.

We recall some further terminology involving partitions and Young diagrams. A skew diagram $\alpha$ is a horizontal strip if it has at most one box in each column; we define the connected components of $\alpha$ by agreeing that two boxes in $\alpha$ are connected if they share a vertex or an edge. If $\mu \subset \lambda$, we let $N(\mu, \lambda)$ denote the number of connected components of $\lambda / \mu$ which do not meet the first column, and we let $N^{\prime}(\mu, \lambda)$ be the one less than the total number of connected components of $\lambda / \mu$. We use the notation $\lambda \backslash \mu$ to denote the partition whose parts are the parts of $\lambda$ which are not parts of $\mu$.

Theorem 3 (Quantum Pieri rule for $L G$ ). For any $\lambda \in \mathcal{D}_{n}$ and $k \geqslant 0$ we have

$$
\sigma_{\lambda} \cdot \sigma_{k}=\sum_{\mu} 2^{N(\lambda, \mu)} \sigma_{\mu}+\sum_{\nu} 2^{N^{\prime}(\nu, \lambda)} \sigma_{\nu} q
$$

in $Q H^{*}(L G(n, 2 n))$, where the first sum is over all strict partitions $\mu \supset \lambda$ with $|\mu|=|\lambda|+k$ such that $\mu / \lambda$ is a horizontal strip, while the second is over all strict $\nu$ contained in $\lambda$ with $|\nu|=|\lambda|+k-n-1$ such that $\lambda / \nu$ is a horizontal strip. 
Proof. The first sum in (7) is just a restatement of the classical Pieri rule of Hiller and Boe [HB]. It follows from Proposition 3 that the coefficient of $q$ in (7) is given by the classical Pieri rule for $L G(n+1,2 n+2)$; one checks easily that the multiplicities agree with those in the theorem (see [KT1, Prop. 7]). Finally, Corollary 4 shows that for all $d \geqslant 2$, the coefficient of $q^{d}$ in $\sigma_{\lambda} \cdot \sigma_{k}$ is zero.

Theorem 4 (Ring presentation and quantum Giambelli). The ring $Q H^{*}(L G)$ is presented as a quotient of the polynomial ring $\mathbb{Z}\left[\sigma_{1}, \ldots, \sigma_{n}, q\right]$ by the relations

$$
\sigma_{i}^{2}+2 \sum_{k=1}^{n-i}(-1)^{k} \sigma_{i+k} \sigma_{i-k}=(-1)^{n-i} \sigma_{2 i-n-1} q
$$

for $1 \leqslant i \leqslant n$, where it is understood that $\sigma_{0}=1$ and $\sigma_{j}=0$ for $j<0$. The Schubert class $\sigma_{\lambda}$ in this presentation is given by the Giambelli formulas

$$
\sigma_{i, j}=\sigma_{i} \sigma_{j}+2 \sum_{k=1}^{n-i}(-1)^{k} \sigma_{i+k} \sigma_{j-k}+(-1)^{n+1-i} \sigma_{i+j-n-1} q
$$

for $i>j>0$, and

$$
\sigma_{\lambda}=\operatorname{Pfaffian}\left[\sigma_{\lambda_{i}, \lambda_{j}}\right]_{1 \leqslant i<j \leqslant r},
$$

where multiplication in $Q H^{*}(L G(n, 2 n))$ is employed throughout and $r$ is the smallest even integer such that $r \geqslant \ell(\lambda)$.

Proof. The quantum relations (8) and the two-condition quantum Giambelli formula (9) are easily deduced from Proposition 3 (following [KT1, §3.3]). We give a new proof of the Pfaffian formula (10).

Suppose $\lambda \in \mathcal{D}_{n}$ with $\ell(\lambda) \geqslant 3$. To prove (10), it suffices to establish the following identity in $Q H^{*}(L G(n, 2 n))$ :

$$
\sigma_{\lambda}=\sum_{j=1}^{r-1}(-1)^{j-1} \sigma_{\lambda_{j}, \lambda_{r}} \sigma_{\lambda \backslash\left\{\lambda_{j}, \lambda_{r}\right\}} .
$$

The classical component of (11) is the classical Pfaffian Giambelli identity for $L G$, as given by Pragacz [P. Hence, (11) is equivalent to

$$
0=\sum_{j=1}^{r-1}(-1)^{j-1}\left\langle\sigma_{\lambda_{j}, \lambda_{r}}, \sigma_{\lambda \backslash\left\{\lambda_{j}, \lambda_{r}\right\}}, \sigma_{\mu}\right\rangle_{d}
$$

for every $d \geqslant 1$ and $\mu \in \mathcal{D}_{n}$ such that $|\lambda|+|\mu|=h(n, d)$. By Corollary [3, the vanishing in (12) will follow from the relations

$$
\sum_{j=1}^{r-1}(-1)^{j-1}\left[X_{\lambda_{j}, \lambda_{r}}^{(1)}\right]\left[X_{\lambda \backslash\left\{\lambda_{j}, \lambda_{r}\right\}}^{(1)}\right]=0
$$

and

$$
\sum_{j=1}^{r-1}(-1)^{j-1}\left[X_{\lambda_{j}, \lambda_{r}}^{(2)}\right]\left[X_{\lambda \backslash\left\{\lambda_{j}, \lambda_{r}\right\}}^{(2)}\right]=0
$$

in $H^{*}(I G(n-1,2 n), \mathbb{Z})$ and $H^{*}(I G(n-2,2 n), \mathbb{Z})$, respectively.

The cohomological formulas (13) and (14) were both proved in [KT1, Cor. 1] by an algebraic argument which established more: namely, that they hold on the level of symplectic Schubert polynomials. The validity of precisely these two identities 
was suggested by the analysis of the Lagrangian Quot scheme in [KT1]. We refer to op. cit. for more details.

Remark 3. We have given independent proofs of the quantum Pieri formula and the quantum Giambelli formula. However, it suffices to prove either of these two results; the other follows formally. In [KT1, the quantum Giambelli formula is proved using intersection theory, and the quantum Pieri formula is deduced by an algebraic argument. For the reverse implication, one may argue as follows. The first relation (9) is derived easily from (7), so we prove the Pfaffian formula (10). We employ the algebra of $\widetilde{Q}$-polynomials $[\mathrm{PR}$. Each $\widetilde{Q}$-polynomial is indexed by a (not necessarily strict) partition $\lambda$, and there are integers $e(\lambda, \mu ; \nu)$ such that

$$
\widetilde{Q}_{\lambda} \widetilde{Q}_{\mu}=\sum e(\lambda, \mu ; \nu) \widetilde{Q}_{\nu}
$$

One now shows by induction on the length $\ell(\mu)$ that

$$
\sigma_{\lambda} \cdot \sigma_{\mu}=\sum 2^{-d} e\left(\lambda, \mu ;\left((n+1)^{d}, \nu\right)\right) \sigma_{\nu} q^{d},
$$

using the quantum Pieri rule and the Pieri formula for $\widetilde{Q}$-polynomials ( $\underline{\mathrm{PR}}$, Prop. 4.9]) as the base case. The quantum Giambelli formula (10) is recovered by combining (15D) with the Pfaffian relations

$$
\widetilde{Q}_{\lambda}=\sum_{j=1}^{r-1}(-1)^{j-1} \widetilde{Q}_{\lambda_{j}, \lambda_{r}} \cdot \widetilde{Q}_{\lambda \backslash\left\{\lambda_{j}, \lambda_{r}\right\}} .
$$

\section{The orthogonal Grassmannian $O G(n+1,2 n+2)$}

4.1. Preliminaries. In this section we give an analogous treatment of the even orthogonal Grassmannian $O G=O G(n+1,2 n+2)=S O_{2 n+2} / P_{n+1}$. This variety parametrizes (one component of) the locus of maximal isotropic subspaces of a $(2 n+2)$-dimensional vector space $E$, equipped with a nondegenerate symmetric form. Recall that $O G$ is isomorphic to the odd orthogonal Grassmannian $S O_{2 n+1} / P_{n}$, hence the results of this section apply to the latter variety as well. The Schubert varieties $X_{\lambda}\left(F_{\bullet}\right)$ in $O G$ are again parametrized by partitions $\lambda \in \mathcal{D}_{n}$ and defined by the equations (4), with respect to a complete isotropic flag $F_{\text {. }}$ in $E$. Let $\tau_{\lambda}$ be the cohomology class of $X_{\lambda}\left(F_{\bullet}\right)$; the set $\left\{\tau_{\lambda} \mid \lambda \in \mathcal{D}_{n}\right\}$ is a $\mathbb{Z}$-basis of $H^{*}(O G, \mathbb{Z})$. For each $k \geqslant 0$, let $O G(k, 2 n+2)$ denote the orthogonal Grassmannian parametrizing $k$-dimensional isotropic subspaces of $E$.

As before, the degree of a morphism $f: \mathbb{P}^{1} \rightarrow O G$ is defined as the degree of $f_{*}\left[\mathbb{P}^{1}\right] \cdot \tau_{1}$. We say that two points $U, V$ of $O G(e, 2 e)$ are in general position if the corresponding subspaces have trivial intersection; the parity condition $\operatorname{dim}(U \cap V) \equiv$ $e(\bmod 2)$ shows that this can happen only if $e$ is even. Proposition 2 has the following orthogonal analogue.

Proposition 4. Let $U, V$, and $W$ be three points of $O G(e, 2 e)$ in pairwise general position (so, in particular, $e=2 d$ is even). Then there is a unique morphism $f: \mathbb{P}^{1} \rightarrow O G(e, 2 e)$ of degree d such that $f(0)=U, f(1)=V$, and $f(\infty)=W$.

An important difference between this and the previous section is that the natural embedding of $O G(n+1,2 n+2)$ into the type $A$ Grassmannian $G(n+1,2 n+2)$ is degree-doubling. This means that for any degree $d$ map $f: \mathbb{P}^{1} \rightarrow O G$, the pullback of the tautological quotient bundle over $O G$ has degree $2 d$. Moreover, the dimension of $\operatorname{Ker}(f)$ is bounded below by $n+1-2 d$. 
4.2. Main result for $O G(n+1,2 n+2)$. Let $Y_{d}$ denote the orthogonal Grassmannian $O G(n+1-2 d, 2 n+2)$. For a given $d$ with $0 \leqslant d \leqslant(n+1) / 2$ and subvariety $\mathcal{W} \subset O G(n+1,2 n+2)$, define $\mathcal{W}^{(d)} \subset Y_{d}$ by the same prescription (5) as in type $C$. For each Schubert variety $X_{\lambda}=X_{\lambda}\left(F_{\bullet}\right)$ in $O G$, we thus get a Schubert variety $X_{\lambda}^{(d)}=X_{\lambda}^{(d)}\left(F_{\bullet}\right)$ in $Y_{d}$. We note that for $d=1, X_{\lambda}^{(1)}$ is the variety which was denoted by $\mathfrak{Y}_{\lambda}$ in [KT2, $\left.\S 3.1\right]$.

Let $h^{\prime}(n, d)=n(n+1) / 2+2 n d$. The Gromov-Witten invariant $\left\langle\tau_{\lambda}, \tau_{\mu}, \tau_{\nu}\right\rangle_{d}$ is zero unless the dimension condition $|\lambda|+|\mu|+|\nu|=h^{\prime}(n, d)$ is satisfied, and this occurs only for $d \leqslant(n+1) / 2$. We now have the following orthogonal analogue of Theorem 2] which is used to identify Gromov-Witten invariants on $O G(n+1,2 n+2)$ with classical structure constants on non-maximal orthogonal Grassmannians.

Theorem 5. Let $d$ be an integer and $\lambda, \mu, \nu \in \mathcal{D}_{n}$ be such that $|\lambda|+|\mu|+|\nu|=$ $h^{\prime}(n, d)$, and let $F_{\bullet}, G_{\bullet}$, and $H_{\bullet}$ be complete isotropic flags of $E=\mathbb{C}^{2 n+2}$ in general position. Then the map $f \mapsto \operatorname{Ker}(f)$ gives a bijection of the set of rational maps $f: \mathbb{P}^{1} \rightarrow O G(n+1,2 n+2)$ of degree d satisfying $f(0) \in X_{\lambda}\left(F_{\bullet}\right), f(1) \in X_{\mu}\left(G_{\bullet}\right)$, and $f(\infty) \in X_{\nu}\left(H_{\bullet}\right)$, with the set of points in the intersection $X_{\lambda}^{(d)}\left(F_{\bullet}\right) \cap X_{\mu}^{(d)}\left(G_{\bullet}\right) \cap$ $X_{\nu}^{(d)}\left(H_{\bullet}\right)$ in $Y_{d}=O G(n+1-2 d, 2 n+2)$.

Corollary 5. Let $d \geqslant 0$ and choose $\lambda, \mu, \nu \in \mathcal{D}_{n}$ with $|\lambda|+|\mu|+|\nu|=h^{\prime}(n, d)$. We then have

$$
\left\langle\tau_{\lambda}, \tau_{\mu}, \tau_{\nu}\right\rangle_{d}=\int_{O G(n+1-2 d, 2 n+2)}\left[X_{\lambda}^{(d)}\right] \cdot\left[X_{\mu}^{(d)}\right] \cdot\left[X_{\nu}^{(d)}\right] .
$$

Corollary 6. In the situation of Corollary 5, if any of $\lambda, \mu$, and $\nu$ has length less than $2 d-1$, then the Gromov-Witten invariant $\left\langle\tau_{\lambda}, \tau_{\mu}, \tau_{\nu}\right\rangle_{d}$ vanishes.

The proofs of these results are similar to those of Theorem 2 and its corollaries. This time the auxiliary space $Y^{\prime \prime}$ is the isotropic partial flag variety $O G(n+1-2 d$, $n+3-2 d ; 2 n+2)$, and we invoke Proposition 4. Note that a stronger version of the vanishing statement in Corollary 6] was established in [KT2 Thm. 6].

4.3. Quantum cohomology of $O G(n+1,2 n+2)$. The quantum cohomology ring of $O G(n+1,2 n+2)$ is defined in a similar way to that of $L G$. A big difference here is that the degree of $q$ is $2 n$. We now give independent proofs of the basic structure theorems [KT2, Thm. 1] and [KT2, Cor. 5] regarding the quantum cohomology of $O G$.

Theorem 6 (Quantum Pieri rule for $O G$ ). For any $\lambda \in \mathcal{D}_{n}$ and $k \geqslant 0$ we have

$$
\tau_{\lambda} \cdot \tau_{k}=\sum_{\mu} 2^{N^{\prime}(\lambda, \mu)} \tau_{\mu}+\sum_{\nu} 2^{N^{\prime}(\lambda, \nu)} \tau_{\nu \backslash(n, n)} q,
$$

where the first sum is over all strict $\mu \supset \lambda$ with $|\mu|=|\lambda|+k$ such that $\mu / \lambda$ is a horizontal strip, and the second sum is over all partitions $\nu=(n, n, \bar{\nu})$ with $\bar{\nu}$ strict such that $\nu \supset \lambda,|\nu|=|\lambda|+k$, and $\nu / \lambda$ is a horizontal strip.

Proof. The argument here differs from that in the proof of Theorem 3 , We first show that $\tau_{\lambda} \cdot \tau_{k}$ is classical whenever $\lambda_{1}<n$; in other words, if the first row of $\lambda$ is not full, then multiplying $\tau_{\lambda}$ by a special Schubert class carries no quantum correction. Observe that Corollary [6 implies $\left\langle\tau_{\lambda}, \tau_{k}, \tau_{\mu}\right\rangle_{d}=0$ for $d>1$.

Suppose now that some Gromov-Witten invariant $\left\langle\tau_{\lambda}, \tau_{k}, \tau_{\mu}\right\rangle_{1}$ is non-zero. It follows that for some complete isotropic flags $F_{\bullet}, G_{\bullet}$, and $H_{\bullet}$, there are some (only 
finitely many) lines on $O G$ incident to $X_{\lambda}\left(F_{\bullet}\right), X_{k}\left(G_{\bullet}\right)$, and $X_{\mu}\left(H_{\bullet}\right)$. One sees easily that given a point $U \in X_{\lambda}\left(F_{\bullet}\right)$, with $\lambda_{1}<n$, there exists a flag $F_{\text {. }}^{\prime}$ such that $U \in X_{\rho_{n-1}}\left(F_{\bullet}^{\prime}\right) \subset X_{\lambda}\left(F_{\bullet}\right)$, where $\rho_{n-1}=(n-1, \ldots, 2,1)$. Moreover, for any $V \in X_{k}\left(G_{\bullet}\right)$, there exists a flag $G_{\bullet}^{\prime}$ such that $V \in X_{n}\left(G_{\bullet}^{\prime}\right) \subset X_{k}\left(G_{\bullet}\right)$.

We claim now that whenever there is one line on $O G$ incident to $X_{\rho_{n-1}}\left(F_{\bullet}\right)$, $X_{n}\left(G_{\bullet}\right)$, and the point $W$, there are infinitely many lines satisfying these incidence conditions. In light of this, it is impossible to have $\left\langle\tau_{\lambda}, \tau_{k}, \tau_{\mu}\right\rangle_{1} \neq 0$. To prove the claim, we recall that lines on $O G$ are in bijective correspondence with points in $O G(n-1,2 n+2)$, and we translate the given incidence conditions to conditions on the corresponding $(n-1)$-dimensional isotropic space $T$ :

(i) the line meets $X_{n}\left(G_{\bullet}\right)$ if and only if $T \subset G_{1}^{\perp}$;

(ii) the line meets $X_{\rho_{n-1}}\left(F_{\bullet}\right)$ if and only if $\operatorname{dim}\left(T \cap \widetilde{F}_{n+1}\right) \geqslant n-2$, where $\widetilde{F}_{n+1}$ is the unique maximal isotropic subspace containing $F_{n}$ other than $F_{n+1}$;

(iii) the line meets the point $W$ if and only if $T \subset W$.

Assume that $T$ satisfies (i)-(iii). Then

$$
\operatorname{dim}\left(G_{1}^{\perp} \cap \widetilde{F}_{n+1} \cap W\right) \geqslant n-2 .
$$

Consider a fixed subspace $S \subset G_{1}^{\perp} \cap \widetilde{F}_{n+1} \cap W$, with $\operatorname{dim} S=n-2$. Now any $T^{\prime}$ of dimension $n-1$ containing $S$ and contained in $G_{1}^{\perp} \cap W$ satisfies (i)-(iii) as well, and there are infinitely many such $T^{\prime}$.

Next, consider any product $\tau_{\lambda} \cdot \tau_{k}$. The first sum in (16) agrees with the classical Pieri formula, so we focus on the quantum term. When $\lambda_{1}<n$, the quantum term (16) correctly vanishes, so we suppose $\lambda_{1}=n$. We write $\lambda \backslash n=\left(\lambda_{2}, \lambda_{3}, \ldots\right)$; we then have the equation

$$
\tau_{\lambda}=\tau_{n} \cdot \tau_{\lambda \backslash n}
$$

in $Q H^{*}(O G)$.

Suppose, first, that $k=n$. Note that the formula $\tau_{n}^{2}=q$ holds in $Q H^{*}(O G)$; this follows from the easy enumerative fact that there is a unique line in $O G$ through a given point and incident to $X_{n}\left(F_{\bullet}\right)$ and $X_{n}\left(G_{\bullet}\right)$, for general flags $F_{\bullet}$ and $G_{\bullet}$. Therefore, in this case, we have $\tau_{k} \cdot \tau_{\lambda}=\tau_{n}^{2} \tau_{\lambda \backslash n}=q \tau_{\lambda \backslash n}$, and the quantum Pieri formula is verified. If $k<n$, then we write

$$
\tau_{k} \cdot \tau_{\lambda}=\tau_{n} \cdot\left(\tau_{k} \cdot \tau_{\lambda \backslash n}\right) .
$$

The product in parentheses receives no quantum correction, and hence is given by the classical Pieri formula. As the quantum Pieri formula for multiplication by $\tau_{n}$ has already been established, it remains only to verify that the result agrees with (16), and this is easily checked.

Theorem 7 (Ring presentation and quantum Giambelli). The ring $Q H^{*}(O G)$ is presented as a quotient of the polynomial ring $\mathbb{Z}\left[\tau_{1}, \ldots, \tau_{n}, q\right]$ modulo the relations

$$
\tau_{i}^{2}+2 \sum_{k=1}^{i-1}(-1)^{k} \tau_{i+k} \tau_{i-k}+(-1)^{i} \tau_{2 i}=0
$$

for all $i<n$, together with the quantum relation

$$
\tau_{n}^{2}=q
$$


(it is understood that $\tau_{j}=0$ for $j>n$ ). The Schubert class $\tau_{\lambda}$ in this presentation is given by the Giambelli formulas

$$
\tau_{i, j}=\tau_{i} \tau_{j}+2 \sum_{k=1}^{j-1}(-1)^{k} \tau_{i+k} \tau_{j-k}+(-1)^{j} \tau_{i+j}
$$

for $i>j>0$, and

$$
\tau_{\lambda}=\text { Pfaffian }\left[\tau_{\lambda_{i}, \lambda_{j}}\right]_{1 \leqslant i<j \leqslant r},
$$

where quantum multiplication is employed throughout and $r$ is the smallest even integer such that $r \geqslant \ell(\lambda)$.

Proof. That the ring presentation is as claimed follows from the argument in ST] (see also [KT2, §3.3]). Formula (17) is true classically and holds without any quantum correction for degree reasons, $\operatorname{since} \operatorname{deg}(q)=2 n$. Formula (18) is equivalent to the Pfaffian Laplace-type expansion

$$
\tau_{\lambda}=\sum_{j=1}^{r-1}(-1)^{j-1} \tau_{\lambda_{j}, \lambda_{r}} \tau_{\lambda \backslash\left\{\lambda_{j}, \lambda_{r}\right\}} .
$$

Observe that (19) will follow from Corollary 5 and the formula

$$
\sum_{j=1}^{r-1}(-1)^{j-1}\left[X_{\lambda_{j}, \lambda_{r}}^{(1)}\right]\left[X_{\lambda \backslash\left\{\lambda_{j}, \lambda_{r}\right\}}^{(1)}\right]=0
$$

in $H^{*}(O G(n-1,2 n+2), \mathbb{Z})$. Finally, (20) was established in [KT2, Cor. 1] by an algebraic argument, whose motivation was similar to that in the Lagrangian case.

At the end of $\$ 3.3$ we noted that it suffices to prove either the quantum Giambelli or quantum Pieri formula; the other then follows using the algebra of symmetric polynomials. The same remark applies to $O G$, and the details are similar (one of the two implications was worked out in [KT2, Sec. 6]).

\section{REFERENCES}

[BCM] L. Bégin, C. Cummins and P. Mathieu : Generating-function method for fusion rules, J. Math. Phys. 41 (2000), no. 11, 7640-7674. MR 2001m:17032

[BKMW] L. Bégin, A. N. Kirillov, P. Mathieu and M. A. Walton : Berenstein-Zelevinsky triangles, elementary couplings, and fusion rules, Lett. Math. Phys. 28 (1993), no. 4, 257-268. MR 94g:81159

[BMW] L. Bégin, P. Mathieu and M. A. Walton : $\widehat{\operatorname{su}}(3)_{k}$ fusion coefficients, Modern Phys. Lett. A 7 (1992), no. 35, 3255-3265. MR 93j:81028

[BS] N. Bergeron and F. Sottile : Schubert polynomials, the Bruhat order, and the geometry of flag manifolds, Duke Math. J. 95 (1998), no. 2, 373-423. MR 2000d:05127

[Be] A. Bertram : Quantum Schubert calculus, Adv. Math. 128 (1997), no. 2, 289-305. MR 98j:14067

[BCF] A. Bertram, I. Ciocan-Fontanine and W. Fulton : Quantum multiplication of Schur polynomials, J. Algebra 219 (1999), no. 2, 728-746. MR 2000k:14042

[Bu1] A. S. Buch : Quantum cohomology of Grassmannians, Compositio Math., to appear.

[Bu2] A. S. Buch : A direct proof of the quantum version of Monk's formula, Proc. Amer. Math. Soc., to appear.

[BKT1] A. S. Buch, A. Kresch, and H. Tamvakis : Grassmannians, two-step flags, and puzzles, in preparation.

[BKT2] A. S. Buch, A. Kresch, and H. Tamvakis : Quantum Pieri rules for isotropic Grassmannians, in preparation. 
[FK] S. Fomin and A. N. Kirillov : Quadratic algebras, Dunkl elements, and Schubert calculus, Advances in geometry, pp. 147-182, Progr. Math. 172, Birkhäuser Boston, Boston, MA, 1999. MR 2001a:05152

[F] W. Fulton : Young tableaux, L.M.S. Student Texts 35, Cambridge Univ. Press, Cambridge, 1997. MR 99f:05119

[FP] W. Fulton and R. Pandharipande: Notes on stable maps and quantum cohomology, in: Algebraic geometry (Santa Cruz, 1995), Proc. Sympos. Pure. Math. 62, Part 2, Amer. Math. Soc., Providence (1997), pp. 45-96. MR 98m:14025

[HB] H. Hiller and B. Boe : Pieri formula for $S O_{2 n+1} / U_{n}$ and $S p_{n} / U_{n}$, Adv. Math. 62 (1986), 49-67. MR 87k:14058

[K] A. Knutson : Private communication.

[KTW] A. Knutson, T. Tao and C. Woodward : The honeycomb model of $G L(n)$ tensor products II: Puzzles determine facets of the Littlewood-Richardson cone, J. Amer. Math. Soc., to appear.

[KT1] A. Kresch and H. Tamvakis : Quantum cohomology of the Lagrangian Grassmannian, J. Algebraic Geom., to appear.

[KT2] A. Kresch and H. Tamvakis : Quantum cohomology of orthogonal Grassmannians, Compositio Math., to appear.

[P] P. Pragacz : Algebro-geometric applications of Schur S-and Q-polynomials, Séminare d'Algèbre Dubreil-Malliavin 1989-1990, Lecture Notes in Math. 1478, 130-191, Springer-Verlag, Berlin, 1991. MR 93h:05170

[PR] P. Pragacz and J. Ratajski : Formulas for Lagrangian and orthogonal degeneracy loci; $\widetilde{Q}$-polynomial approach, Compositio Math. 107 (1997), no. 1, 11-87. MR 98g:14063

[ST] B. Siebert and G. Tian : On quantum cohomology rings of Fano manifolds and a formula of Vafa and Intriligator, Asian J. Math. 1 (1997), no. 4, 679-695. MR 99d:14060

[S] F. Sottile : Pieri's formula for flag manifolds and Schubert polynomials, Ann. Inst. Fourier (Grenoble) 46 (1996), no. 1, 89-110. MR 97g:14035

[Tu] G. Tudose : On the combinatorics of sl(n)-fusion coefficients, preprint (2001).

[Y] A. Yong : Degree bounds in quantum Schubert calculus, Proc. Amer. Math. Soc., to appear.

Matematisk Institut, Aarhus Universitet, Ny Munkegade, 8000 Århus C, Denmark

E-mail address: abuch@imf.au.dk

Department of Mathematics, University of Pennsylvania, 209 South 33rd Street, Philadelphia, Pennsylvania 19104-6395

E-mail address: kresch@math.upenn.edu

Department of Mathematics, Brandeis University - MS 050, P. O. Box 9110, Waltham, MASSACHUSETTS 02454-9110

E-mail address: harryt@brandeis.edu 